\title{
Gravitational wave background discovered by NANOGrav as evidence of a cyclic universe
}

\author{
Gorkavyi N. ${ }^{\mathrm{a}}$ \\ ${ }^{a}$ Science Systems and Applications, Inc., 10210 Greenbelt Road, Lanham, MD 20706, US
}

\begin{abstract}
The consortium NANOGrav discovered the isotropic gravitational wave background (GWB) with an amplitude of $h \sim 10^{-15}$ and a frequency of $f \sim 10^{-8}$ $\mathrm{Hz}$ using observations of millisecond pulsars. We hypothesize that the GWB is relic radiation left over from the merging stellar mass black holes (SBHs) during Big Crunch at $z \sim 10^{10}$. The relic gravitational waves are similar to the gravitational waves with $f \sim 10^{2} \mathrm{~Hz}$ discovered by LIGO in 2015, taking into account a decrease in frequency by a factor $\sim 10^{10}$ due to the expansion of the Universe. We take as a basis the observed spectrum of $139 \mathrm{SBHs}$, discovered by the LIGO observatory. Our model explains well all the observed features of the GWB. Unlike all other GWB models, our model predicts a sharp decrease in the GWB amplitude at frequencies $f>3.5 * 10^{-8} \mathrm{~Hz}$, reflecting the deficit of SBHs with masses $<4 M_{\odot}$. The SBMH mergers at Big Crunch should generate yet undiscovered GWB with a frequency of $f \sim 10^{-(14-17)} \mathrm{Hz}$.

Keywords: Gravitational waves, NANOGrav, black holes, oscillating Universe 2010 MSC: 83-57, 83-35
\end{abstract}

\section{Highlights}

- Gravitational Wave Background (GWB) is relic radiation left over Big Crunch at $z \sim 10^{10}$

- The relic gravitational waves are similar to the gravitational waves discovered by LIGO 
- Decrease in $10^{10}$ times the frequency of GWB is associated with the expansion of the Universe.

- The model predicts a sharp decrease in the GWB amplitude at frequencies $f>3.5 * 10^{-8} \mathrm{~Hz}$,

\section{Introduction}

Isotropic CMB radiation discovered by [1] is relic radiation of a compressed hot Universe, which decreased its temperature during its expansion [2, 3, 4. The relic gravitational waves (GW) were discussed in many papers (see, for example, [5, 6]). The most powerful known sources of gravitational radiation are merging black holes (BH). In 2015, the LIGO group discovered gravitational waves of $f \sim 10^{2} \mathrm{~Hz}$ caused by the merging of black holes with stellar masses (SBH up to 100 solar masses) [7]. Gravitational radiation from modern binary SMBH can generate gravitational waves with a length of about a light year, which can be detected by variations in the radio pulses from millisecond pulsars $8,0,10$. Recently, the NANOGrav consortium announced the possible discovery of an isotropic stochastic background of gravitational waves with a frequency $f \sim$ $2.5 * 10^{-9} \div 1.2 * 10^{-8} \mathrm{~Hz}$ (or $2.5 \div 12 \mathrm{nHz}$ ) and an amplitude of $h \sim 10^{-15}$ [11]. It is assumed that these waves are generated by the current binary SMBH [10, although many other hypotheses of origin the GWB have been put forward: from collapsing fluctuations in the early stages of the Big Bang [12, 13] to the break of a cosmic string [14.

Our work analyzes the formation of the GWB in a cyclic Universe. The model of an oscillating Universe was popular until the 1980s [2, 3, 5, 15] and now there is a growing interest in this model [16, 17, 18, 19. The classical cyclic cosmology included a mechanism of the periodical transformation of chemical elements: the effective photodissociation of heavy atomic nuclei into individual

baryons began when the temperature of the electromagnetic relic radiation in the compressed Universe reached $\sim 3 * 10^{10} \mathrm{~K}$. This is how hydrogen was created to 
form stars in a new cycle. The temperature $3 * 10^{10} \mathrm{~K}$ is reached at $z \sim 1.6 * 10^{10}$ corresponds to the radius of the Universe $\approx 3$ ly $[20$.

In [19, 21, 22, a cyclic model of the Universe with a large number of black holes has been analyzed (see Fig. 1). Obviously, when the Universe collapses, a significant part of the gravitational mass of the merging black holes turns into gravitational waves. The Big Bang mechanism in such a model is based on the Schwarzschild metric with variable mass 22,24 . The era of multiple merger of black holes begins when the Universe shrinks to a volume comparable to the total volume of existing BHs. This process should be natural for many cyclic models of the Universe [16, 17, 19, 21, 22, 25]. It is easy to estimate that the total volume of $\mathrm{BHs}$ in the Universe is determined by the most massive SMBHs: if we take the average SMBH mass of $10^{8} M_{\odot}$ [12, then $10^{11}$ such holes can be packed into a box with a size of 0.3 ly. If the $\mathrm{SMBH}$ mass is $10^{9} M_{\odot}$ [12, then even $10^{9}$ such holes will require a box with a size of $0.7 \mathrm{ly}$. Thus, when the size of the contracting Universe decreases by $\sim 10^{10}$ times - up to several light years, the black holes will begin to merge en masse due to lack of space. There is an equilibrium value $z_{0}$ that allows oscillations of the Universe, which does not accumulate heavy elements and is consistent with the observed population of black holes. If $z<z_{0}$ then the temperature of the Universe will be insufficient for photodissociation of iron nuclei. If $z>z_{0}$ then the total volume of observed black holes will be too large for such a small volume of the Universe. In 22] the estimate $z_{0} \sim 10^{10}$ was obtained independently of the data of NANOGrav.

We hypothesize that the long-wavelength gravitational radiation recorded by NANOGrav [11] is the relic gravitational radiation from the multiple merger of black holes during the Big Crunch: $f_{G W B} \sim z_{0}^{-1} f_{L I G O}$. Consequently, GWB is a fossil version of GW discovered by LIGO and stretched due to the expansion of the Universe. Let us show that the NANOGrav data can be explained within the framework of a cyclic model of the Universe with dark matter from black holes without putting forward new hypotheses or fitting free parameters.

It is also assumed by [26, 27] that the GWB detected by NANOGrav are generated during the formation of SBHs in the early stages of the Universe. In 

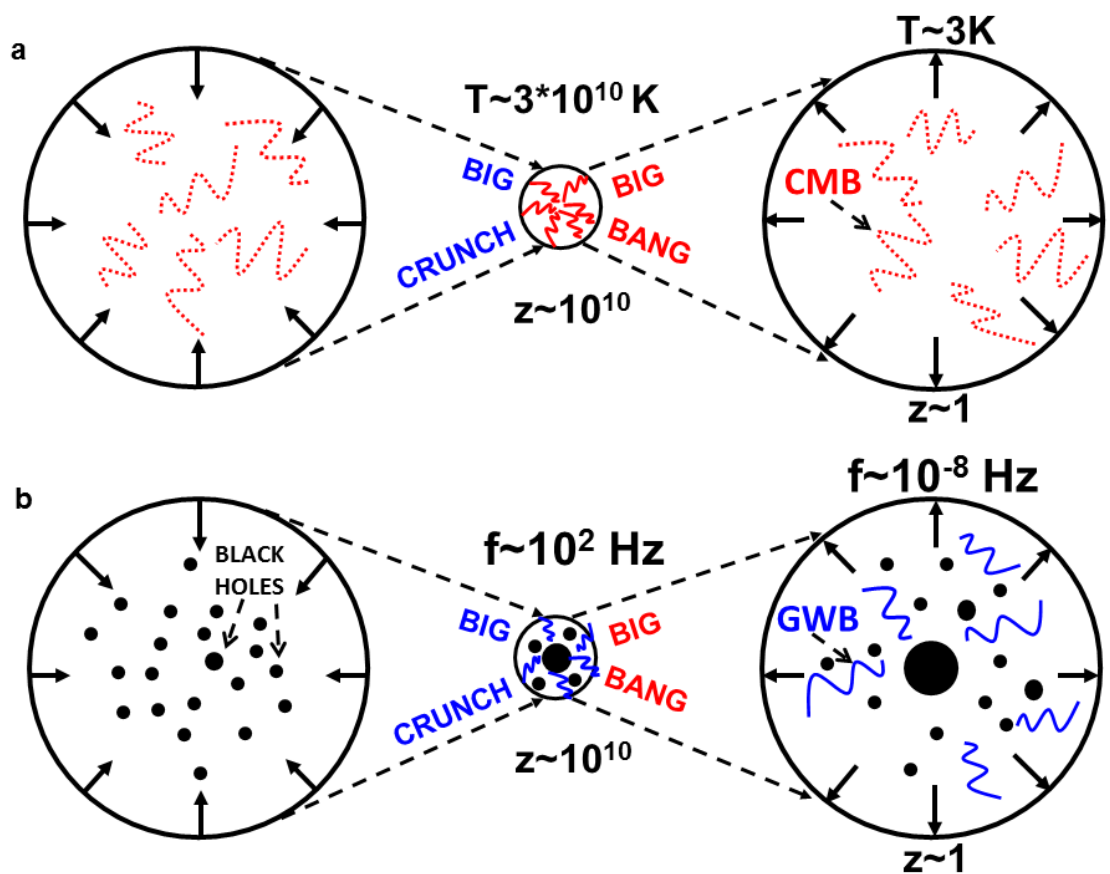

Figure 1: (From 22], with modifications). Evolution of relict electromagnetic waves (CMB) (a) and gravitational radiation (GWB) (b) in the Universe during Big Crunch and Big Bang.

our model these SBHs come from the past cycles of the Universe. As will be shown below, our model and the hypothesis published by [26, 27] have important differences in observational predictions.

\section{GWB in a one-component model}

Let us evaluate the modern frequencies and amplitudes of GWs, which were formed during Big Crunch. The equation for the amplitude of gravitational radiation from a binary system of black holes of the same mass $M$, located at a distance D from the observer, can be written as [10]:

$$
h=4 \sqrt{\frac{2}{5}}\left(\frac{G M}{c^{3}}\right)^{5 / 3}\left(\frac{2 \pi}{T}\right)^{2 / 3} \frac{c}{D},
$$

where $T$ is the proper rotation period of the binary system. Formula (1) and subsequent formulas are obtained in the case of asymptotically flat spacetime. 
In the case of a closed Universe, the formulas for length and mass include the factor $\left(1-\left(D / R_{U}\right)^{2}\right)^{-1 / 2}$, where $R_{U}$ is the radius of curvature of the Universe (see, for example, [15). We will use formula (1) and subsequent formulas, assuming that the discussed distances are less than the radius of curvature of the Universe $\left(D / R_{U}\right)^{2}<<1$. The characteristic time of gravitational radiation of such a system can be obtained from the equation [10]:

$$
\frac{1}{\tau}=\frac{96}{5}\left(\frac{G M}{c^{3}}\right)^{5 / 3}\left(\frac{2 \pi}{T}\right)^{8 / 3}
$$

The amplitude and frequency of the gravitational wave reach their maximum value and the period $T$ minimum value just before the merger of black holes. For this case, equations (1) and (2) are simplified. The maximum frequency of gravitational radiation when two identical black holes merge is written in the form (see, for example, [28]):

$$
f=\frac{2}{T(1+z)}=\frac{c}{12 \sqrt{6} \pi R(1+z)} \approx \frac{2.2 * 10^{3}}{M_{s}(1+z)}
$$

where $M_{s}$ is the mass of the black hole in solar masses, and $f$ is measured in hertz. The value $(1+z)$ in the denominator describes the decrease in frequency due to the cosmological redshift, and the coefficient 2 in the numerator appears due to the quadrupole nature of gravitational radiation [11]. From (3) we obtain that the merger of black holes with a mass of $\sim 10 M_{\odot}$ (just such events were registered by LIGO [7]) gives the natural frequency of the generated gravitational waves $f_{0}=2 / T \approx 2.2 * 10^{2} \mathrm{~Hz}$. Taking into account (3) and $z \sim 1.6 * 10^{10}$, we obtain the observed frequency of the gravitational stochastic radiation $f \sim 1.4 * 10^{-8} \mathrm{~Hz}$, which coincides with the frequency of the waves discovered by NANOGrav. We obtain from (3) the minimum value of $T$ :

$$
T=\frac{24 \sqrt{6} \pi R}{c} .
$$

and substitute (4) in (1), taking into account the equation for the radius of the black hole $R=2 G M / c^{2}$. We get:

$$
h=\frac{1}{3} \sqrt{\frac{2}{5}} \frac{R}{D}
$$


For merging two $\mathrm{BH}$ with $R \approx 10^{7} \mathrm{~cm}$ and a distance $D \sim 10^{9}$ ly, we get typical amplitude of GW, registered by LIGO: $h \sim 10^{-21}$ [7]. Equation [2] is also simplified for the case of maximum frequency (3):

$$
\frac{1}{\tau}=\frac{4}{135} \frac{c}{R}
$$

To estimate the total energy of gravitational radiation from $N$ merging black holes, we will square the amplitude (5) and write:

$$
h^{2}=\frac{2}{45}\left(\frac{R}{D}\right)^{2} N \frac{\tau}{t_{0}},
$$

where $t_{0}$ is the free path time of a $\mathrm{BH}$ moving at a speed $V$, before collision and merging with another $\mathrm{BH}$ [29]:

$$
\frac{1}{t_{0}}=108 \pi R^{2} n V
$$

where $n=3 N /\left(4 \pi a^{2}\right)$ is the concentration of black holes located in a sphere with radius $a$. The ratio $\tau / t_{0}$ characterizes the fraction of time during which a hole emits, merging with another hole. From Eq. (7), taking into account Eq. (6) and Eq. (8), we obtain for the average amplitude of background gravitational waves generated by SBH during Big Crunch:

$$
h \approx 9 \sqrt{\frac{3}{2}} \frac{N R^{5 / 2}}{D a^{3 / 2}} \sqrt{\frac{V}{c}}
$$

where for the estimation it was taken: $N=10^{22}[19] ;=10^{7} \mathrm{~cm} ; D \approx 10^{10}$ ly; $a \approx 3$ ly; $V \approx c$. We assumed that the relative velocities of black holes in a maximally compressed Universe are comparable to the speed of light. The hypothesis about the accumulation of black holes and GWB from cycle to cycle of the Universe [19] will change the meaning of the parameters in Eq. 99, for example, $N$ will mean the sum of GWB sources for a certain number of cycles. Let's compare the amplitudes of the background gravitational waves from the SBH merger at Big Crunch $z \sim 10^{10}$ and from the SMBH merger at a later time (at $z$ of the order of unity):

$$
\frac{h_{S B H}}{h_{S M B H}} \approx\left(\frac{R_{S B H}}{R_{S M B H}}\right)^{5 / 2}\left(\frac{a_{S M B H}}{a_{S B H}}\right)^{3 / 2} \frac{N_{S B H}}{N_{S M B H}} A \sim 10^{11}
$$


Table 1: Estimates of the amplitude and frequency of the GWB from different sources

\begin{tabular}{|c|c|c|}
\hline Source and $z$ & Big Crunch, $z \sim 10^{10}$ & $z \sim 1$ \\
\hline SBH & $h \sim 10^{-15}, f \sim 10^{-8} H z$ & $h \sim 10^{-30}, f \sim 10^{2} \mathrm{~Hz}$ \\
SMBH & $h \sim 10^{-11}, f \sim 10^{-14} \mathrm{~Hz}$ & $h \sim 10^{-26}, f \sim 10^{-4} \mathrm{~Hz}$ \\
\hline
\end{tabular}

The following estimates were accepted here:

$$
\frac{R_{S B H}}{R_{S M B H}} \sim 10^{-6} ; \frac{a_{S M B H}}{a_{S B H}} \sim 10^{10} ; \frac{N_{S B H}}{N_{S M B H}} \sim 10^{11}
$$

The last factor in 10 is insignificant, because it is close enough to 1 :

$$
A=\frac{D_{S M B H}}{D_{S B H}} \sqrt{\frac{V_{S B H}}{V_{S M B H}}} \sim 1
$$

We can obtain estimates of the background waves generated by black holes at different stages of the evolution of the Universe (see Table 1, where the mass of a SMBH is $10^{6}$ times the mass of an SBH).

It follows from 10 and Table 1 that the amplitude of the stochastic gravitational waves caused by the SBH merger at the Big Crunch stage significantly exceeds the amplitude of the waves generated by modern SMBH mergers at the centers of galaxies. Note that estimates of emission from modern SMBHs usually consider not a moment of merger, but rather stable orbital of black holes around the center of mass [10]. In this case, the radiation amplitudes of the individual system are less, but the radiation time $\tau$ in 7 is longer. Unfortunately, there is a large uncertainty in such calculations, associated with the probability of merging galaxies and the problem of approaching the central SMBHs inside the last parsecs [10, 28, 30]. Merging during Big Crunch SMBH with mass $\sim 10^{7} \div 10^{10} M \odot$ will generate radiation with a frequency of $f_{0} \sim 10^{-(4 \div 7)} \mathrm{Hz}$. Taking into account $z \sim 10^{10} \mathrm{~Hz}$, we get that in the modern epoch there should be very long gravitational waves with a frequency $f \sim 10^{-(14 \div 17)} \mathrm{Hz}$. Such waves have a length of $\sim 10^{-(1 \div 4)}$ of the size of the Universe. It follows from (9) that the SMBH fusion will generate a GWB with a significant amplitude. For example, for a frequency $f \sim 10^{-14} \mathrm{~Hz}$, the GW amplitude will be $\sim 10^{4}$ 
times greater than the amplitude of GW discovered by NANOGrav [11] (see Table 11). The discovery of such wavelengths is possible using data from the Gaia astrometric satellite [31, 32, 33].

\section{GWB in a multi-component model}

In our estimates, we assumed that all BHs have the same mass. Let us assume that the mass distribution of SBHs participating in mergers during Big Crunch is close to the modern distribution of black holes because a population of relic black holes can form from astrophysical black holes that accumulate in the repeating cycles of the Universe [19. The observed population of black holes consists mainly of SBH from 4 to $100 M \odot$ [19, 34] and SMBH with $10^{5}-10^{10} M \odot$ located in the centers of galaxies [35].

It should be taken into account that formula $(9)$ includes the mass distribution of black holes $N(m)$. Figure 2 shows the spectrum of mass of $\mathrm{BH}$ as a function of frequency $h(f)$ under the assumption that merging black holes generate gravitational radiation of one wavelength (with maximum frequency and amplitude). Figure 2 shows that the detected gravitational waves are located near the maximum amplitude of the gravitational waves, which follow from our model.

Also Figure 2 shows that due to the influence of the mass spectrum of black holes, the frequency dependence of the amplitude can vary significantly. For $f \approx$ $(2.5 \div 6.3) * 10^{-9} \mathrm{~Hz}$ (or $2.5 \div 6.3 \mathrm{nHz}$ ) our model gives $h \propto f^{-(0.5 \div 0.6)}$, which close to the observation of NANOGrav (see Figure 2). The spectrum of gravitational radiation from small $(4 \div 20 M \odot) \mathrm{SBHs}$ is dependent on observational selection, so it is difficult to draw any reliable conclusions for $f>6.3 * 10^{-9} \mathrm{~Hz}$ (or 6.3 $\mathrm{nHz}$ ). The most reliable observational fact is the absence of black holes less than 4 solar masses $f>3.2 * 10^{-8} \mathrm{~Hz}$ (or $f>32 \mathrm{nHz}$ ), which should be reflected in the spectrum of gravitational waves. The $f=\lg \left(3.2 * 10^{-8}\right)=-7.5$ barrier (marked with a vertical line) corresponds to the period of gravitational waves in one year. According to observations there are no astrophysical black holes 


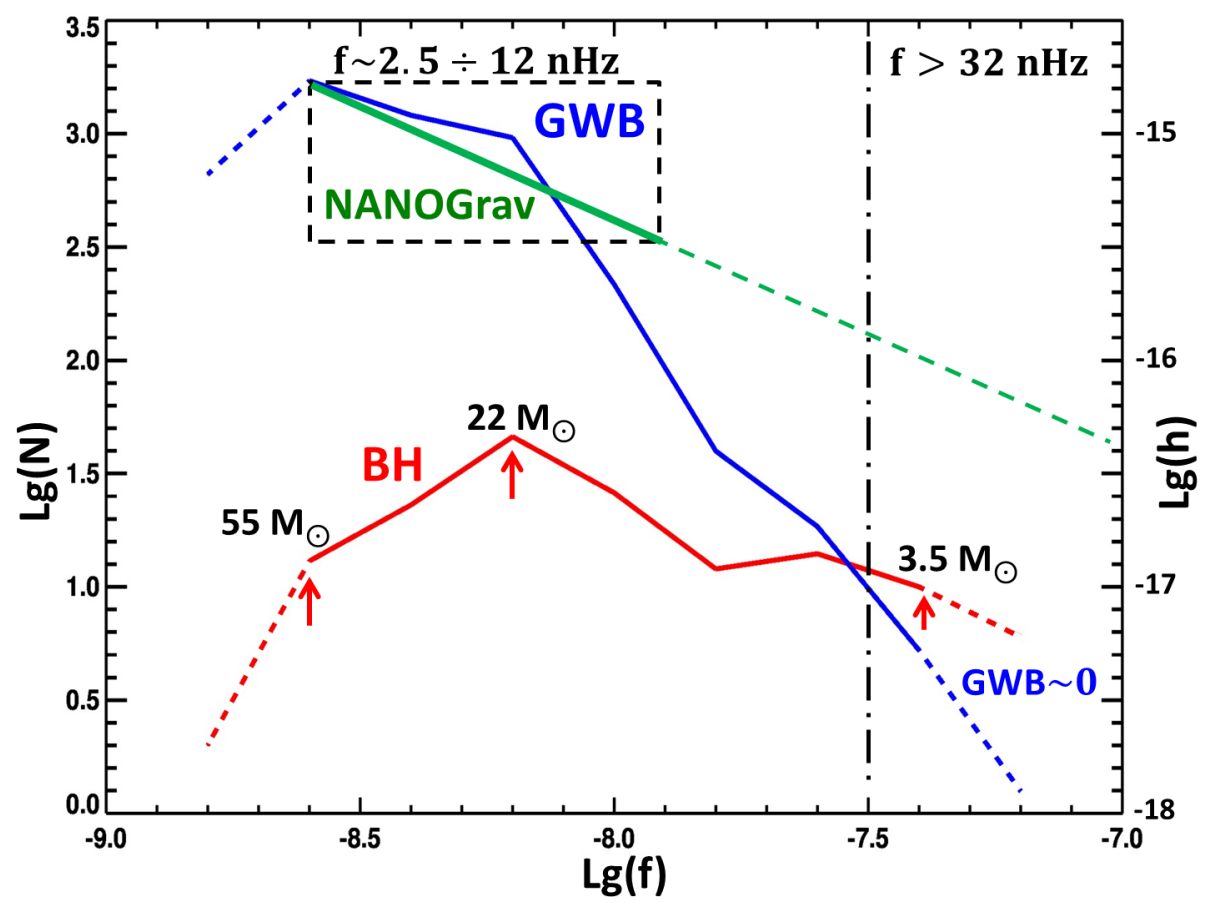

Figure 2: Solid curve BH: mass spectrum of SBH according to LIGO data with the addition of black holes in binary stellar systems [34, 35] as a function of GW frequency (arrows indicate the magnitude of mass of BHs corresponding to the specific frequency of GWB). Solid line GWB shows the theoretically calculated amplitude of gravitational radiation during the SBH merger. Dashed parts of curves BH and GWB show zones with low statistics. The dashed rectangle shows the border corresponding to the frequency of gravitational waves discovered by NANOGrav (thick straight line, the maximum of which is aligned with the maximum of the model curve). The dash-dotted vertical line shows the border corresponding to the frequency of gravitational waves of $3.2 * 10^{-8} \mathrm{~Hz}$ (or 1/year) from the merging of black holes at $\sim 4 M \odot$, so to the right of this line there will be a strong deficit of GWB in comparison with the extrapolated NANOGrav data (dashed continuation of a thick straight line) and theoretical models based on the gravitational waves from SMBHs [10. 
less than 4 times the mass of the Sun, or there are very few of them. This should cause a strong deficiency of GWB with frequencies $f>3.2 * 10^{-8} \mathrm{~Hz}$ (see Figure 2). Indeed, no "any detectable contributions from a GWB" in frequency $f>1.2 * 10^{-8} \mathrm{~Hz}$ (or $12 \mathrm{nHz}$ ) [1]. We consider this fact to be very important. The deficit (beyond the expected power-law dependences) of GWB at frequencies of $f>3.2 * 10^{-8} \mathrm{~Hz}$ (or $f>32 \mathrm{nHz}$ ) will confirm our model.

\section{Discussion}

Stochastic gravitational waves must be accompanied by a quadrupole spatial correlation according to the Hellings-Downs curve [36]. As the results of NANOGrav show, the background of stochastic gravitational waves has no signs of monopole and dipole waves. At the same time, GWB has some signs of quadrupole spatial correlation, but with a low level of statistical confidence [1]. After submitting this article to the journal, the article [37] was published with the results of the analysis of the Parkes Pulsar Timing Array (PPTA). Under the assumptions of an analysis of the NANOGrav, the team of PPTA have detected with high confidence a common-spectrum time-correlated signal in the timing of the 26 PPTA-DR2 millisecond pulsars: $h \sim 2.2 * 10^{-15}$ at a frequency of $1 \mathrm{yr}^{-1}$ 37. The PPTA data also showed statistically weak indications of quadrupole spatial correlation. [38] show that the current lack of evidence for quadrupolar spatial correlations is consistent with the magnitude of the correlation coefficients for pairs of Earth-pulsar baselines in the array, and the fact that pulsar timing arrays are most-likely operating in the intermediate-signal regime. We believe that the weak quadrupole correlation found in both works (Fig.7 [11] and Fig.3 [37]) will increase with the amount of data used. The discussions around the GWB ([11; [37; [38]) show that the statistical methods used in the analysis of pulsar signals contain significant assumptions that must be examined to finally prove the validity of the discovery. From our point of view, the reliability of the discovery of the stochastic background of gravitational waves is confirmed by the fact that the existence of a powerful background of 
nanohertz relict waves was predicted in the article [19], submitted to the journal in May 2020.

\section{Conclusions}

LIGO detectors observe gravitational waves from rare modern mergers of SBHs - the most numerous black holes in the Universe. We believe that the telescopes of the NANOGrav and the PPTA detected a burst of similar gravitational radiation from the numerous $\mathrm{SBH}$ mergers during Big Crunch. Decrease in $10^{10}$ times the frequency of these relic gravitational waves is associated with the expansion of the Universe. We believe that the NANOGrav and the PPTA data support the hypothesis that black holes can make up the bulk of dark matter [39, 40, 41] and that a significant portion of the observed black holes came from the past cycle of the oscillating Universe [16, 42. We find it remarkable that the parameter $z \sim 10^{10}$, which satisfies the model of a cyclic Universe, turns out to be just such that the frequency of relic gravitational waves coincides with the frequency of gravitational waves observed by NANOGrav and PPTA.

From our model, specific conclusions can be drawn that can be verified by observations:

1. The spectrum of GWB discovered by NANOGrav and PPTA will not be described by Planck's law or any other law related to thermal equilibrium. The GWB should reflect the observed distribution of black holes (SBHs and SMBHs).

2. The amplitude of GWB discovered by NANOGrav and PPTA decreases with increasing frequency, but the rate of this decrease will become especially noticeable at frequency $f>3.2 * 10^{-8} \mathrm{~Hz}$ (or $f>32 \mathrm{nHz}$ ) (the oscillation period is less than a year) due to a lack of astrophysical black holes with masses $<4 M \odot$. This feature of the spectrum cannot be predicted by any model based on the hypothesis of primordial black holes born in the Big Bang (see, for example, [26]). 
3. The numerous SMBHs that are currently observed in the centers of galaxies should generate low frequency GWB $f \sim 10^{-(14 \div 17)} \mathrm{Hz}$ during the Big Crunch.

\section{Declaration of Competing Interest}

The author declares that he has no known competing financial interests or personal relationships that could have appeared to influence the work reported in this paper.

\section{Acknowledgments}

The author thanks John Mather, Alexander Vasilkov, Sergei Kopeikin and George Hobbs for helpful discussions and comments.

\section{References}

[1] A. Penzias, R. Wilson, Astrophys. J. 142 (1965) 419-421. doi:10.1086/ 148307.

[2] P. Gamov, The Creation of the Universe, Viking Press, New York, 1953.

[3] R. Dicke, P. Peebles, P. Roll, D. Wilkinson, Astrophys. J. 142 (1965) 414419. doi:10.1086/148306.

[4] J. Mather, J. Boslough, The very first light, Basic Books, New York, 2008.

[5] Y. Zel'dovich, I. Novikov, The structure and evolution of the Universe, Univ. of Chicago Press, Chicago and London, 1983.

[6] L. Grishchuk, Physics-Uspekhi 48 (2005) 1235-1247. doi:10.1070/ PU2005V048n12ABEH005795.

[7] B. P. Abbot, R. Abbott, T. D. Abott, et al., Phys. Rev. 116 (2016) 061102. doi:10.1103/PhysRevLett.116.061102. 
[8] M. Sazhin, Soviet Ast. 22 (1978) 36-38.

[9] S. Detweiler, Astrophys. J. 234 (1979) 1100-1104. doi:10.1086/157593.

[10] A. Jaffe, D. Baker, Astrophys. J. 283 (2003) 616-631. doi:10.1086/ 345443.

[11] Z. Arzoumanian, P. Baker, H. Blumer, et al. (The NANOGrav Coll.), Astrophys. J. Letters 905 (2020) L34. doi:10.3847/2041-8213/abd401.

[12] K. Nguyen, T. Bogdanovic, J. Runnoe, et al., Astrophys. J. Letters 905 (2020) L34. doi:10.3847/2041-8213/abd401.

[13] V. D. Luca, G. F. anf A. Riotto, Phys. Rev. Letters 126 (2021) 141303. doi:10.1103/PhysRevLett.126.041303.

[14] W. Buchmuller, V. Domcke, K. Schmitz, Phys. Letters B 811 (2021) 135914. doi:10.1016/j.physletb.2020.135914.

[15] P. Peebles, Principles of Physical Cosmology, Princeton University Press, Princeton, New Jersey, 1993.

[16] V. Gurzadyan, R. Penrose, Eur. Phys. J. Plus 128 (2013) 22. doi:10. 1140/epjp/i2013-13022-4.

[17] N. Poplawski, Gen. Relativ. Gravit. 53 (2021) 18. doi:doi:10.1007/ s10714-021-02790-7.

[18] E. D. Valentino, A. Melchiorri, J. Silk, Nature Astronomy 4 (2019) 196-203. doi:10.1038/s41550-019-0906-9.

[19] N. Gorkavyi, S. Tyul'bashev, Astrophysical Bulletin 76 (2021) 229-247. doi:10.1134/S199034132103007X.

[20] S. Weinberg, Gravitation and Cosmology, Wiley, New York, 1972.

[21] N. Gorkavyi, A. Vasilkov, MNRAS 476 (2018) 1384-1389. doi:10.1093/ mnras/sty335 
[22] N. Gorkavyi, A. Vasilkov, J. Mather, In Exploring the Dark Side of the Universe, https://pos.sissa.it/335/039/pdf, poS(EDSU2018)039 (2018). doi: $10.22323 / 1.335 .0039$

[23] M. Kutschera, MNRAS 345 (2003) L1. doi:10.1046/j.1365-8711.2003. $07045 . \mathrm{x}$

[24] N. Gorkavyi, A. Vasilkov, MNRAS 461 (2016) 2929-2933. doi:10.1093/ mnras/stw1517.

[25] R. Penrose, Cycles of Times, Alfred A. Knopf Publ., New York, 2011.

[26] K. Kohri, T. Terada, Phys. Letters B 811 (2021) 136040. doi:10.1016/j. physletb.2020.136040.

[27] V. Vaskonen, H. Veermae, Phys. Rev. Letters 811 (2021) 051303. doi: 10.1103/PhysRevLett.126.05130.

[28] J. H. Z.L. Wen, F.S. Liu, Astrophys. J. 692 (2009) 511-521. doi:10.1088/ $0004-637 \mathrm{X} / 692 / 1 / 511$

[29] V. Frolov, I. Novikov, The structure and evolution of the Universe, Springer, Netherlands, 1998.

[30] A. Goulding, K. Pardo, J. Greene, et al., Astrophys. J. Letters 879 (2019) L21. doi:10.3847/2041-8213/ab2a14.

[31] C. Gwinn, T. Eubanks, T. Pyne, et al., Astrophys. J. 485 (2019) 87-91. doi:10.1086/304424

[32] L. Book, E. Flanagan, Phys. Rev. D 83 (2011) 024024. doi:10.1103/ PhysRevD.83.024024

[33] C. Moore, D. Mihaylov, A. Lasenby, G. Gilmore, Phys. Rev. Letters 119 (2017) 261102. doi:10.1103/PhysRevLett.119.261102. 
[34] R. Abbot, T. Abbott, S. Abraham, et al. (GWTC-2), Compact binary coalescences observed by ligo and virgo during the first half of the third observing run, https://arxiv.org/abs/2010.14527 (2020).

[35] A. M. Cherepashchuk, Physics-Uspekhi 57 (2014) 359-376. doi:10.3367/ UFNe.0184.201404d.0387.

[36] R. Hellings, G. Downs, Astrophys. J. Letters 265 (1983) L39-L42. doi: $10.1086 / 183954$

[37] B. Goncharov, R. Shannon, D. Reardon, et al., Astrophys. J. Letters 917 (2021) L19. doi:10.3847/2041-8213/ac17f4

[38] J. Romano, J. Hazboun, X. Siemens, et al., Phys. Rev. D 103 (2021) 063027. doi:10.1103/PhysRevD.103.063027.

[39] S. Bird, I. Cholis, J. B. Munoz, et al., Phys. Rev. Lett 116 (2016) 201301. doi:10.1103/PhysRevLett.116.201301.

[40] A. Kashlinsky, Astrophys. J. 823 (2016) L25. doi:10.3847/2041-8205/ $823 / 2 / \mathrm{L} 25$.

[41] S. Clesse, J. Garcia-Bellido, Physics of Dark Universe 15 (2017) 142-147. doi:10.1016/J.DARK.2016.10.002

[42] T. Clifton, B. J. Carr, A. Coley, Class.Quant.Grav. 34 (2017) 135005. doi: $10.1088 / 1361-6382 / \mathrm{aa} 6 \mathrm{dbb}$. 